DOI 10.32370/IA_2019_01_6

\title{
Digital Painting: Creation Artwork in a Graphic Editor
}

\author{
Perova Olga \\ Master of Engineering and Technology
}

\begin{abstract}
With the proliferation of the Internet and the advent of new technologies, many modern artists have begun to give preference to a computer, and work exclusively in graphic editors. The process of changing the traditional tools of the artist, such as an easel, brush and paints, to a graphic tablet is being actively pursued. It is early to say that traditional art is being supplanted by digital, but the trend has already been outlined.

Digital painting is not significantly different from traditional painting. Only the tools have changed, but the laws of composition and color rendering remain the same. It is fair to say that the use of modern technologies has facilitated the implementation of some tasks, significantly accelerated the process of drawing.

The author examines some of the features of work in digital editors, describes the techniques for constructing composition, head and body drawing. In addition, parses the design features of the image of characters in various styles.
\end{abstract}

\section{Construction and drawing of a head with the proportional relations method}

In order to achieve mastery in one of the main sections of the academic drawing - the image of the human head - the artist must study a number of basic rules concerning the anatomical structure, the laws of perspective and the design of the form. But, despite the general foundation of knowledge, drawing "from life" and drawing "according to the imagination" have some differences. An artist working in kind has the ability to compare his drawing and the object depicted, analyze the result and correct mistakes; drawing by imagination is deprived of such a possibility [3].

However, for drawing by imagination, there are methods for constructing a human head, based on the ratio of the proportions of its various parts. These proportional canons were defined in antiquity, and the underlying laws are still true.

To work on the head pattern, it is useful to know the name of the main parts of the skull: 1.Frontal 2.Parietal 3.Temporal 4.Sphenoid 5.Zygoma 6.Maxilla 7.Mandible 8.Occipital 9.Nasal bone (Fig. 1). 


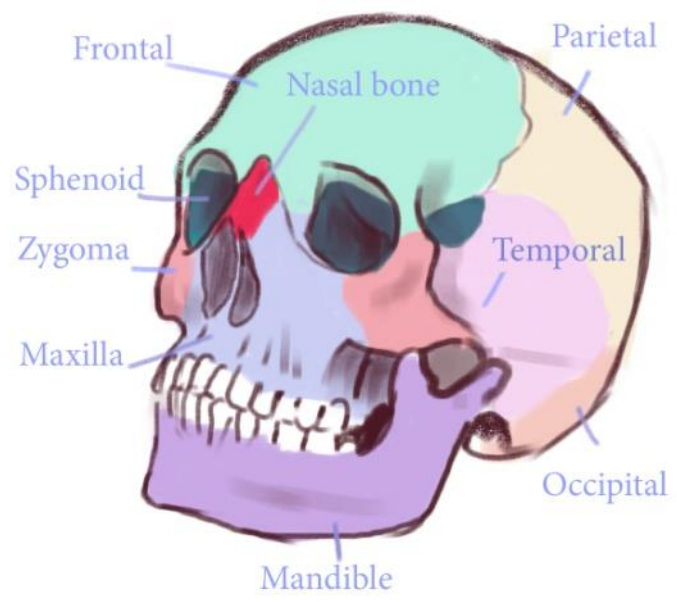

Fig. 1. The main parts of the skull.

In general, the human head can be described as an "oval". Its spatial design is formed by two geometric figures: a sphere - a skull, and a polygonal block that simulates the jaw and cheekbones. Cutting the sides of the sphere, the circle should be divided into four equal parts, of which the vertical line is the beginning of the jaw line and the horizontal line is the line of the eyebrows (Fig. 2).
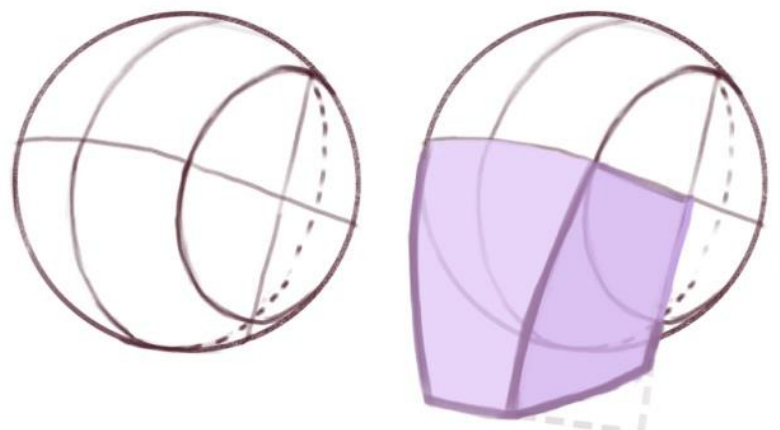

Fig. 2. The division of the sphere into parts

Placing your head in simple shapes, you can easily imagine its position in space. It is necessary to draw a rectangular parallelepiped at the desired angle, dividing it into three equal horizontal parts. The parallelepiped is a kind of container within which a sphere truncated from both sides and a polygonal jaw block will fit. In this construction, the upper line corresponds to the line of the eyebrows, the middle line to the nose, the lower line to the end of the jaw (Fig. 3).

After the artist determines the position of the depicted head in space, he can begin to draw a face using the proportional relationship method. 

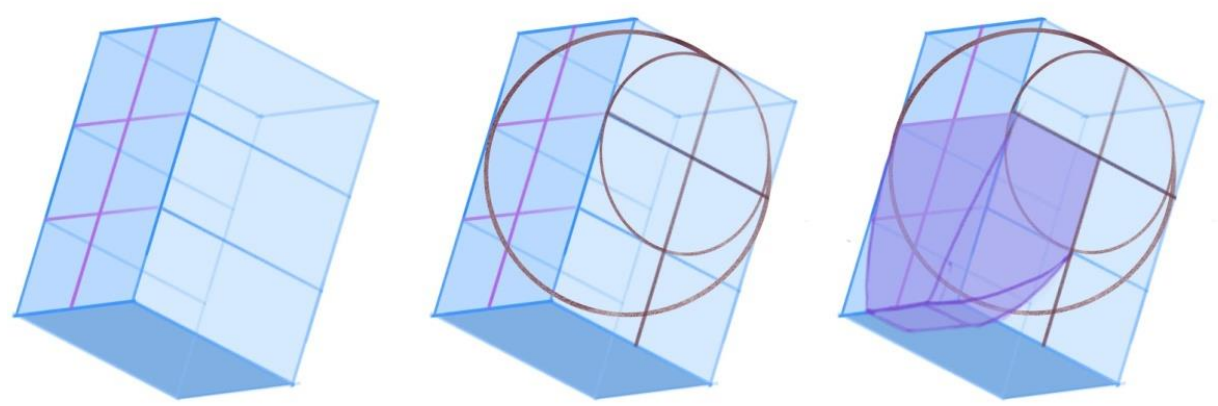

Fig. 3. Construction through simple shapes

The first method: division into three equal parts.

First you need to draw an oval in the shape of an egg. (a) Then divide it into 3.5 equal parts by horizontal lines, as indicated in the figure. The top line is the hairline. The middle one is the brow line, the bottom line is the tip line of the nose. Next, divide the oval in half by a vertical line. (b)

Mark five equal vertical lines. The distance between the lines is the width of the eye. (c) (Fig. 4).

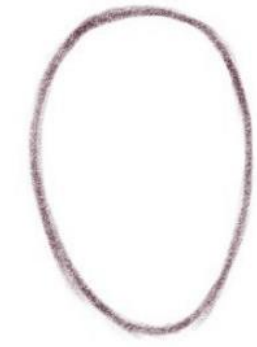

a

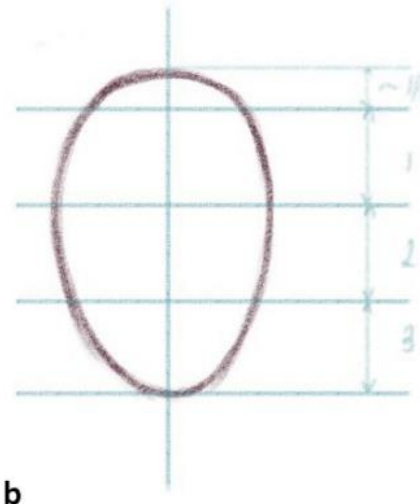

b

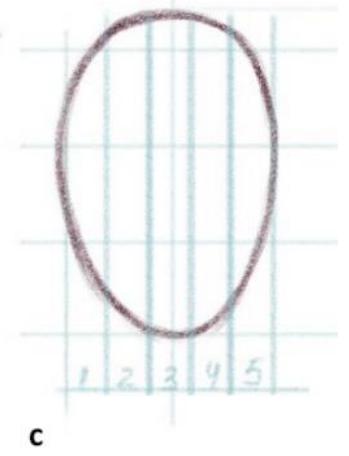

C

Fig. 4. Construction on the first method I.

Now you should sketch out the eyes, eyebrows, nose and mouth. The corners of the mouth correspond to the center of the pupil of the eye. The lower lip lies on the segment number 3. (d) Add parts and mark cheekbones - they start just above the middle of the ear. (e) At the end add shadows and remove construction lines. (f) (Fig. 5).

The second way: the width of the eye as a module. 


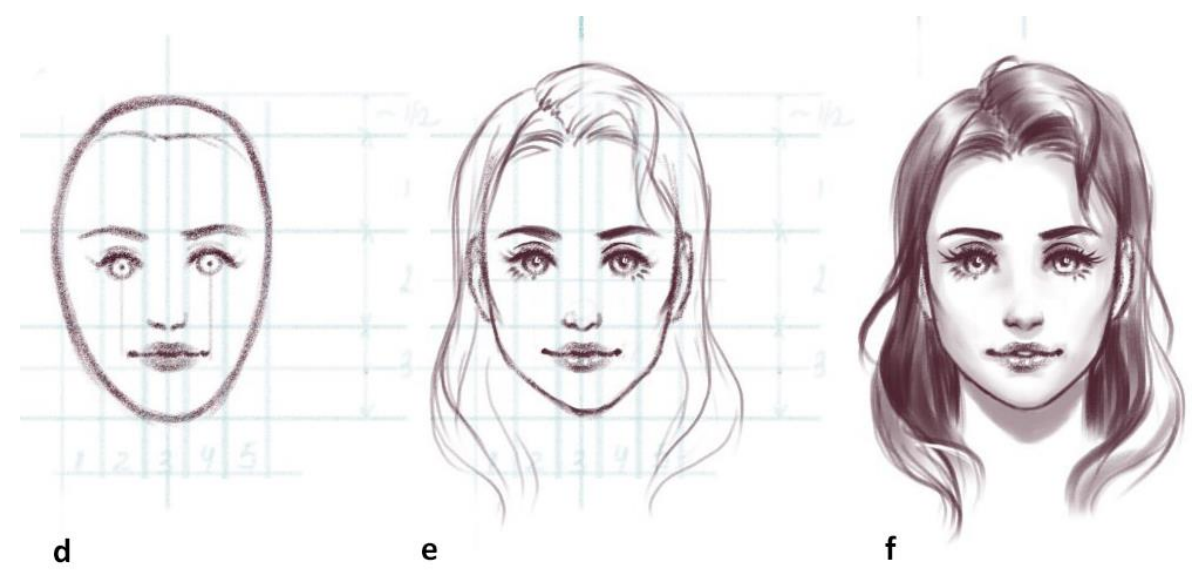

Fig. 5. Construction on the first method II

First you need to draw an oval in the shape of an egg. Divide it into 4 equal parts by vertical and horizontal lines. (a) Similar to the first method, mark five equal vertical lines. The distance between the lines is the width of the eye. (b)

On segments 2 and 4 to depict the eyes. At a distance of one and a half eyes will be located the tip of the nose. The height of the ear is equal to the distance from the tip of the nose to the upper eyelid of the eye. (c) (Fig. 6).

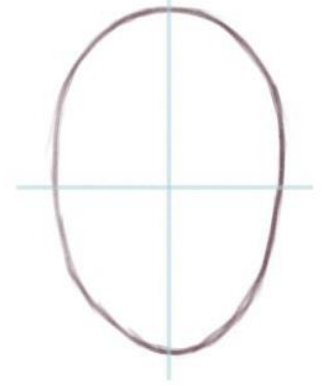

a

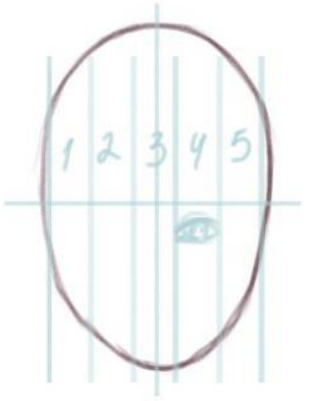

b

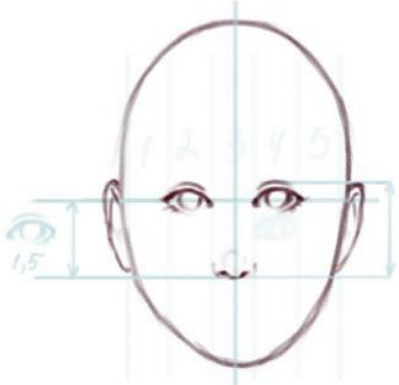

C

Fig. 6. Construction on the second method 1.

Next, mark the lower border of the lips at a distance equal to the width of the eye, measuring it from the tip of the nose. The height of the lips is approximately equal to half of the segment. (d) Add eyelashes, hair and details, falling shadows. (e)

If the work on the picture is carried out in a graphic editor, it is advisable to display the picture horizontally. So you can see the errors: distorted parts of the face and irregularities. Distortion is clearly visible on the displayed figure. (f) This happened because of the not 
absolutely equal shape of the oval. The symmetry of the face should be checked constantly (Fig. 7).

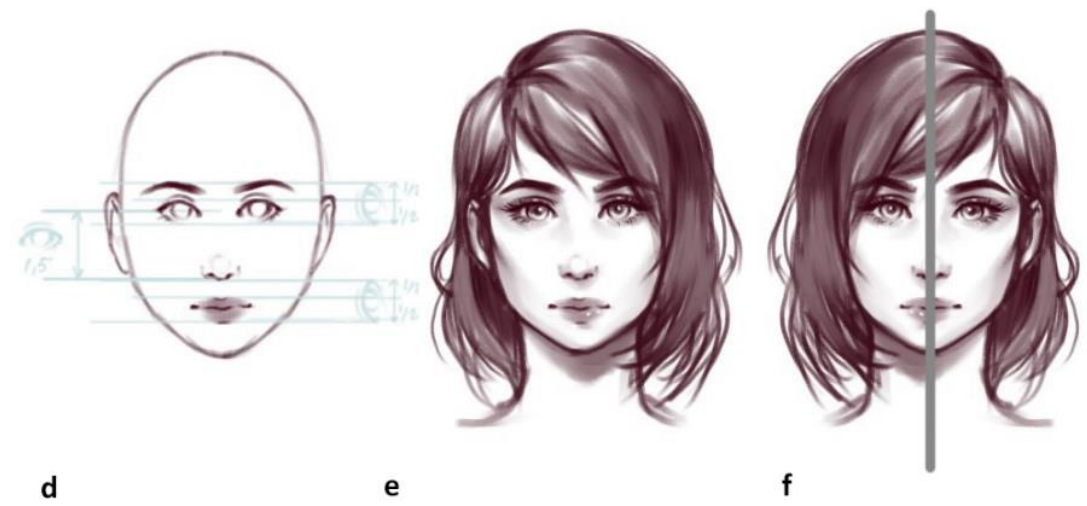

Fig. 7. Construction on the second method II.

It is not recommended to speed up your work by copying one of the parts of the face until the artist has enough experience. You need to try to correct the errors yourself.

The third method is the fastest and easiest to perform.

You need to draw an oval and divide it into 4 equal parts. The resulting horizontal segments divided in half. Above these points will be located the centers of the eyeballs. Next, divide the lower half of the vertical into 5 equal parts. Mark the nose on line number 2 . The lip line will lie on line number 3 (Fig. 8).
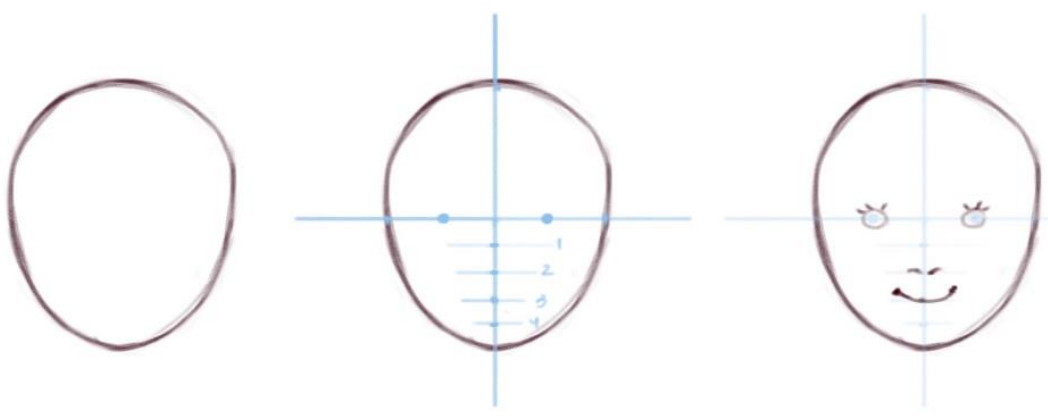

Fig. 8. Construction on the third method I.

Draw on the resulting points the eyes, nose and lips. Outline the ears between the nose line and the upper eyelid of the eye. Display the picture, check it for symmetry and distortion. Finish your eyebrows, eyelashes and lips. Add hair and drop shadows (Fig. 9).

It is necessary to clarify the proportions of a person according to the rule of an equilateral triangle. With the help of a triangle, you can determine the relative position of the eyes and mouth. 

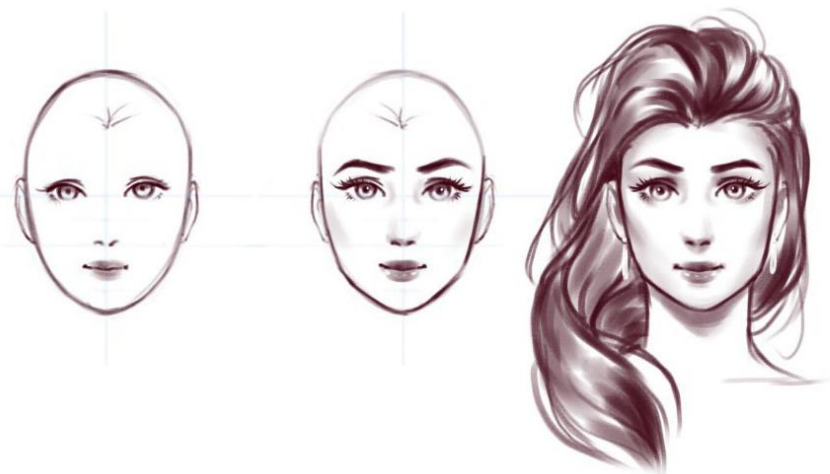

Fig. 9. Construction on the third method II.

The width of the face is about 5 eyes. The lines of the chin and cheeks can also be identified and verified using the eye module (Fig. 10).
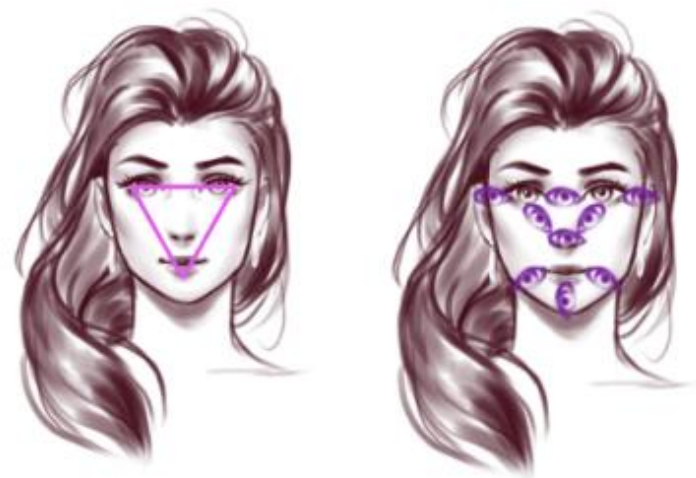

Fig. 10. Construction on the third method III.

Thus, using the method of proportional ratios, the artist can always control the accuracy and accuracy of the head drawing. Due to its simplicity and accessibility, the method is suitable for both specialists and beginners.

\section{Drawing character in different art styles}

Hand-drawn animation (animation) appeared at the end of the 19th century and was most developed in the first half of the 20th century, with the advent of the animated films of Walt Disney. In 1936, the Soyuzmultfilm studio was founded in the USSR, in 1952 the first French cartoon full-length cartoon "Shepherd and the Chimney Sweeper" was released, and in 1958 Japan created its own unique style of hand-drawn animation - anime.

With the spread of animation, the ways of drawing characters developed and developed, acquiring characteristic features. Various schools of animation borrowed artistic techniques from each other, reinterpreting them according to their vision and embodying them in a new, unlike anything else. To date, there is a fairly extensive list of ways to depict characters, each 
of which can be attributed to a particular animation style at a glance [5]. Work with the proportions of the human body, the anatomical features of the structure and the manner of execution of the eyes have become the hallmarks of the art of depicting characters. One of the most recognizable styles is the work of the Disney studio and the Japanese characters of anime films.

Disney style was formed in the 1920s, when Walt Disney founded his studio. In 1937, the full-length animated film "Snow White and the Seven Dwarfs" was released, bringing tremendous success to the creator and laying down the traditions of classic Disney princesses.

It is by the example of one of them, the Little Mermaid, that will further be considered ways of depicting characters in the style of Disney and in the style of anime.

The proportions of the body of Disney characters are generally similar to the proportions of an ordinary human body, but, as a rule, are somewhat shortened in height. For an average person, the height of the head is about seven - seven and a half times it fits in the height of the body, while at Disney this figure decreases to six. The head itself is larger than that of an ordinary person, a few "childish" species. Neck thin, indistinctly pronounced shoulder girdle. Waist, on the contrary, should be read very clearly, while the hips are strongly narrowed. Legs and all parts of the body should be without obvious muscles. The character has overall softness of the line and a feeling of flexibility. The shape of the face with a pointed chin, the size of the eyes is much larger than human ones (Fig. 11).

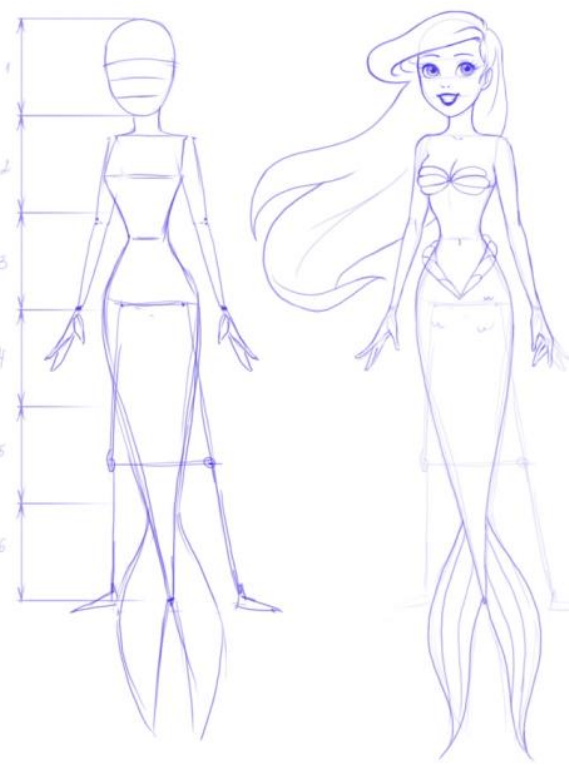

Fig. 11. The proportions of the Disney character.

Eyes are depicted larger, rounded, but with tapering corners. The contour is closed, eyelashes are emphasized by a wide line. On the iris and on the pupil use pure monophonic fillings without effects of multi-layeredness and falling shadows; a highlight is located above (Fig. 12). 


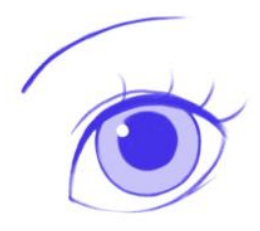

Fig. 12. Eye of the Disney character.

Anime - Japanese animation with a very characteristic character rendering. Contrary to popular belief that the anime in its current form is derived exclusively from Japanese culture, this style arose under the influence of Western animation. The founder of the style Osamu Tezuka was inspired by American cartoons [1]. In particular, the character Betty Boop influenced the appearance of the most remarkable feature of anime characters - a greatly exaggerated eye size. In this case, as a rule, the mouth and nose are shown conditionally.

The proportions of the body are more elongated in comparison with the proportions of the characters of Disney, the height of the head is about seven times in height. The figure is clearly delineated, with pronounced shoulders and developed hips, due to the orientation of the anime - cartoons to a more adult audience. The hair is depicted in large strands, in general, similar to the Disney style. Fingers are represented in sufficient detail and plastically (Fig. 13).

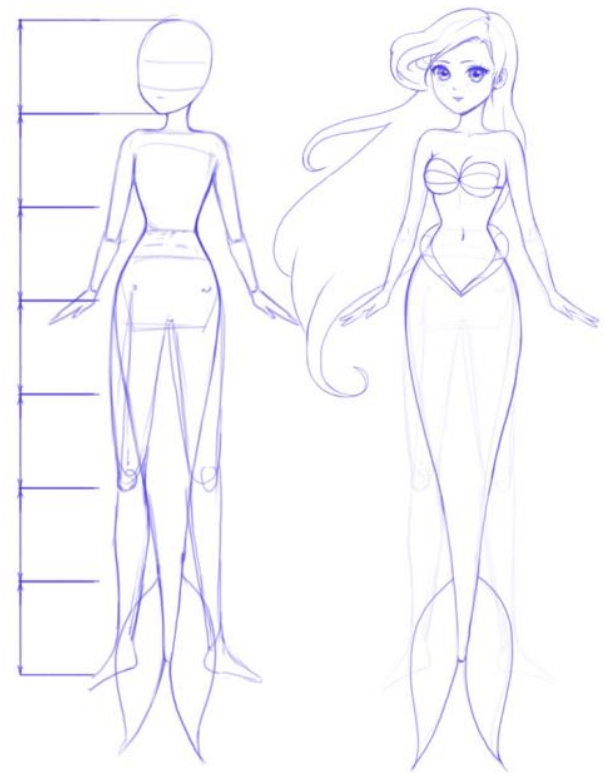

Fig. 13. Anime character proportions.

Unnaturally big eyes are the hallmark of anime characters. They provide basic information about the character as a whole, his age, emotional state, positive or negative character. When drawing, they first of all outline the size of the eye, then designate the upper and lower eyelids, taking into account the almost round shape of the eye. The size of the iris and pupil is determined based on the characteristics of the character - for example, the negative 
or crazy character will have a pupil-point. The contour of the eye is often not closed, the upper lashes are collected in a single volume. The presence of glare is obligatory, often a falling shadow from the upper eyelid is drawn on the surface of the eye (Fig. 14).

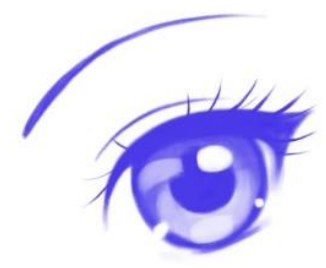

Fig. 14. Eye of anime character.

Chibi is one of the graphic styles of Japanese comics - manga. In anime - cartoons, such characters look deliberately cute, speak in a childish voice, and often have a capricious or nervous character. Characters made in the style of "chibi" have much more pronounced hypertrophied proportions, in comparison with the other styles listed above. Chibi is characterized by a huge head, which occupies $1 / 2$ of the body (the word "chibi" in Japanese means a small person or child). This kind of characters are used to underline the comic image, or for a more vivid expression of emotions, since the "chibi" has large eyes that occupy most of the face.

A chibi character must be small in size, 3 or 4 times smaller than a standard anime character. When constructing a body, two circles should be drawn, of which the upper circle is the size of the head. In the lower circumference, about a third is allocated under the torso, the rest is occupied by the legs. The hands of "chibi" are small and short, fingers are depicted schematically. If the character has his feet, they should be drawn conditionally, in the form of wedge-shaped elements (Fig. 15).
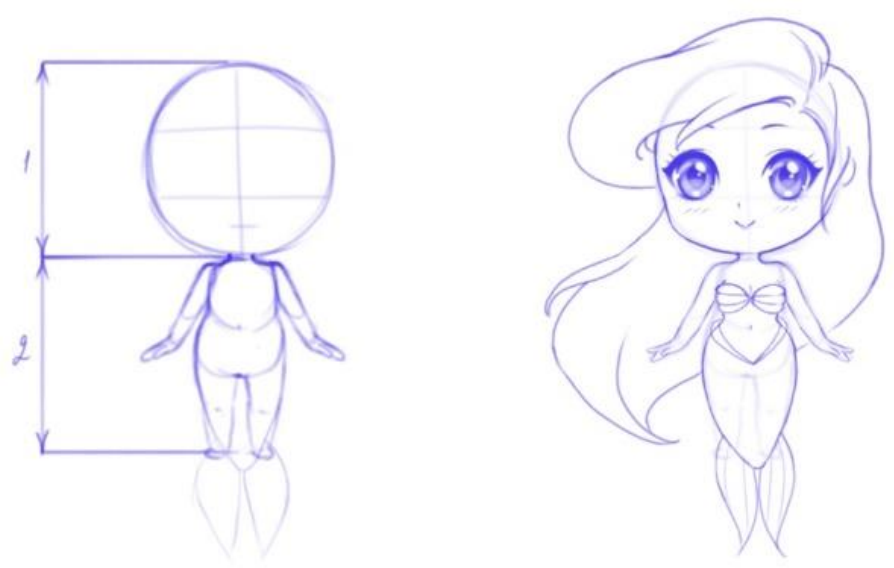

Fig. 15. Character proportions - chibi. 
Despite the large head, chibi characters have a relatively small face with large eyes. The nose is absent, or it is scheduled conditional stroke, the mouth is very small. Eyes - the main and most developed part of the face. The shape is rounded, with a lot of highlights and a falling shadow from the upper eyelid. The main part of the upper eyelashes is drawn in a solid array with a small "arrow" on the outside (if the character is female) (Fig. 16).

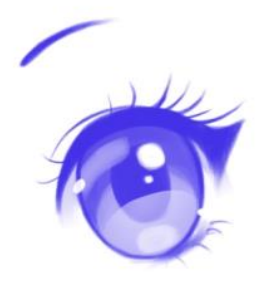

Fig. 16. Eye of chibi-character

It should be noted that the styles of the image of animated characters have long gone beyond the limits of animation. Today, they can be found in computer animation, video games, comics and toys. However, the characteristics remain the same, and knowledge of the basic distinctive features of styles allows the character specialist to work in various fields.

\section{Picturing a person's figure given to the capabilities of modern graphic editors}

In The Treatise on Painting, an Italian artist and scientist Leonardo da Vinci writes that drawing from life should be the basis of the methodology for teaching drawing. So the student studies the characteristics of the structure and shape of the depicted object, carefully observes and reflects. Drawing from nature, according to da Vinci, increases the efficiency of learning and is of interest to the knowledge of life.

The findings of one of the largest representatives of the art of the High Renaissance are relevant to this day and do not disagree with the modern views and attitudes of the academic drawing. However, in those times and now artists are seeking means and ways to speed up, simplify or improve the creative process of drawing.

For example, Leon Battista Alberti, a leading theorist of Renaissance art, one of the founders of the new European architecture, recommended that painters use a curtain of transparent fabric, tracing paper or muslin. The veil was drawn on squares and placed between the eye and the plane of the image, grafted into the same squares. This method was based on the principle of strict observance of the laws of perspective. In order to maintain a single line of the horizon (level of vision) and a vanishing point, a special device was attached to the curtain - a "window of view". In addition, for working outside the workshop, Alberti used a special small camping veil that he could carry around. 
The recognized master of the Western European Renaissance, the German painter and graphic artist Albrecht Dürer wrote: "A Guide to Measurement with a Compass and a Ruler," where he outlined a number of perspective rules. Dürer first proposed using the method of orthogonal projection for perspective design.

The above craftsmen lived and worked in the 15-16 centuries. Obviously, with the advent and mass distribution of computers, many contemporary artists who have switched from traditional painting to digital have incomparably greater technical capabilities.

Graphic editors fully model and copy the traditional tools of the artist. Digital painting is based on the same academic laws as traditional, but, unlike it, almost never involves drawing from life. In the workplace of a digital artist - a computer, graphics tablet, one or two monitors - in principle, does not imply the real presence of the depicted object [4].

As the Renaissance masters sometimes used the devices and devices available to them for more accurate drawing, so the modern artist uses a number of methods of construction.

As an example, consider these methods in relation to the image of a human figure.

\section{- Sketching by photo.}

In the artistic community, this method has an ambiguous reputation and is often presented in a negative way. Drawing does not require the artist's skills in building shapes, knowledge of anatomy and design features of the form. A fair comparison will be made with traditional artists who have been convicted of pre-printing an image on canvas and then putting paint over the print.

However, painting from a photograph is the easiest and most accessible way. Virtually all graphic editors allow you to upload a photo image and work on top of it. The artist becomes available not only the form that he can simply circle, but also the colors of the photo, the lighting, the surroundings.

The artist has access to online photobanks with a huge number of photographs of models in various angles. Some of them are distributed for a fixed price, some can be used for free. If necessary, a specific angle, unavailable in the photobank, the artist can personally make the desired photo, using as a model someone from relatives, friends, or himself in the mirror image.

The artist can also transform a photo image using the built-in graphic editor toolkit. He can increase and decrease the whole image or any part of it, distort the shape, adjust and adjust color and light, tone and saturation.

Despite the ethical aspect described above of using photographs in the artist's work, this technique is more than justified in certain areas where the final image is not presented as a 
unique, independent work. For example, in the production of video game content, in conceptual designs and sketches, where the image of a person directly does not play a decisive role.

- Using a 3D model.

The three-dimensional model replaces the artist's real model. A 3D model, unlike a twodimensional plane photograph, can be unfolded, adjusting its position to the desired angle; High-quality 3D-model has hinged bendable limbs for more fine-tuning. In a sense, the artist works with a flexible dummy capable of taking on any position.

The advantages of this method are the ability to set the model to the correct angle. Finding the right photo sometimes takes an unreasonable amount of time; In addition, even a selected photo image may not be absolutely true to the artist's plan.

After setting up the model is completed, the artist proceeds to drawing. At this stage, he either takes a snapshot of the resulting angle and then works with him as with a photograph, with all the pluses and minuses of this method; or draws an image, looking at a 3D model, imitating a drawing from life.

Probably, working with high-quality 3D-models could be more common, but even in all the multitude of Internet content it is relatively difficult to find them. In addition, the artist may require additional software installed on the computer, regardless of the graphic editor.

- Brushes with the option to draw shapes.

In such graphic editors as Adobe Photoshop, the user can access extensive brush settings. In addition to brushes that imitate watercolor or oil painting, pencil stroke, or even a quill pen, the artist can create a brush that leaves a mark in the shape of a silhouette of a human figure [6].

To do this, some preliminary work should be done, namely, to draw, first of all, directly the figure itself, using any method, including the two described above.

Then, the resulting silhouette figure is attached to a specific brush, with which the artist sets the name, for example, "Brush_figure_1". Then each trace of this brush or, figuratively speaking, each touch of the brush to the canvas will leave a mark in the form of a silhouette of a figure. Of course, there cannot be any particular flexibility of speech - the artist has only a specific, given silhouette with minimal editing options.

This method may seem absurd. However, it is indispensable for digital artists working with the concepts of the environment. It can be concepts for video games, movies and other things.

Suppose an artist needs to paint a not too carefully drawn concept of a fantasy world, where the center of the picture would be the market square of a fairytale city with a crowd of 
people. He will depict the city walls, the square paved with stones and rag stalls, after which he will need to sketch a crowd of hundreds of people.

Instead of manually drawing dozens of shapes, the artist uses his brushes with the option to draw shapes. Having a large set of brushes with figures from several different angles, he quickly places them across the square, changing color, scale, making mirror copies or combining various elements from different shapes.

In this way, the artist will quickly achieve the feeling of extras without extra work.

- Collage.

As with the use of the brush with the option to draw shapes, the collage is more suitable for quick and not very carefully worked out concepts and sketches than for completed fullfledged works of art. In a community of digital artists, a collage is known as photobashing.

At its core, it is similar to the depiction of a photograph. In the collage also used images, but, as a rule, in larger quantities.

The artist working with a collage collects a figure from fragments of various photos. This style does not imply a mandatory stroke and drawing over the photo. The resulting figure in its final form may consist of clearly different fragments, producing a somewhat slovenly impression.

Despite this, the specificity of drawing concepts fully admits a similar style. When a digital artist is faced with the task of creating several concepts during a working day, mainly conveying an idea, a general style and atmosphere, a quick collage, like brushes with the shape drawing parameter, will be the best solution.

\section{Working on a composition in a graphics editor}

Composition - an organizing element of the art form, connecting disparate details into an organic and integral structure. Compositionally verified pattern, in which objects are connected with each other by semantic and spatial arrangement, is perceived as a single product, regardless of the number of objects present on it. This is one of the fundamental foundations of fine art, true not only for traditional, but also for digital painting. Despite the fact that the classical definition of composition appeared almost six hundred years ago in the era of the Italian Renaissance, modern artists working in graphic editors on a computer follow the same compositional rules. At the same time, they have incomparably large technical capabilities - a leap in the development of information technologies has opened new methods and techniques in the field of visual arts. In particular, raster graphic editors (like Adobe Photoshop) have a functional that allows you to easily transform and modify a drawing at any stage, which has a very positive effect on the early stage of working with composition [2]. 
Before you begin work on the construction of the composition, you should carefully consider the content of the future picture and its semantic content. The artist needs to decide whether he wants to see his picture static and balanced, or, on the contrary, convey movement through a dynamic composition. It is also necessary to clearly identify the central element of the picture, the so-called compositional center, and to focus on it.

For precise positioning of elements on a virtual canvas, you should use guide lines with their help, you can quickly outline a composition grid, convenient for working with the "rule of thirds", according to which the sheet is divided into 9 parts using 2 parallel horizontal and 2 vertical lines. In such a grid, objects are located, as a rule, at the intersection of lines, or on the line itself (Fig. 17a).

In a similar way, a grid of guides can be used to build a composition according to the golden section, spiral (Fig. 17b) or diagonal (Fig. 17c) rule (Adobe Photoshop graphics editor does not have diagonal guides, you will have to draw them manually).

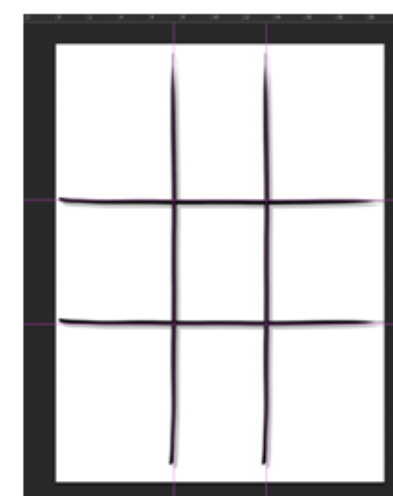

a

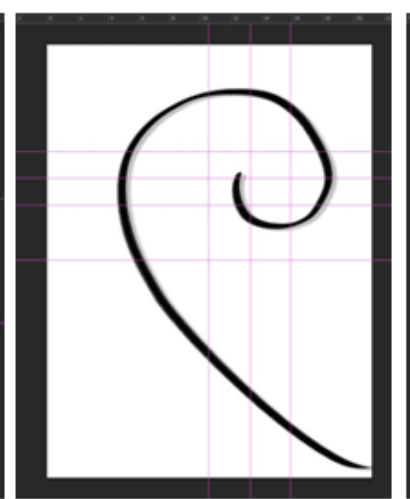

b

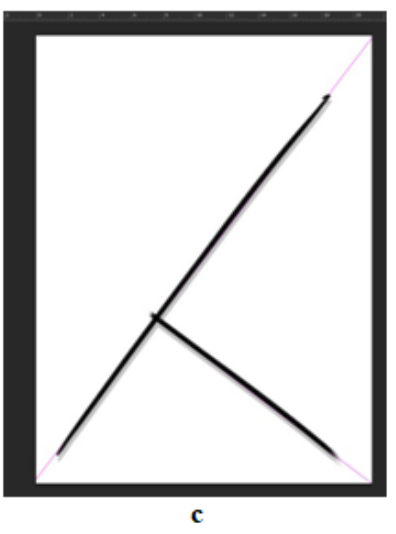

Fig. 17. Construction of a composite grid along the guides: a) rule of third; b) spiral golden section; c) diagonal golden section.

Next comes the work directly on the composition. As a rule, several quick sketches are performed with the search for the most successful option. After this option is chosen, the artist can carry out an additional step of finalizing the composition using the tools of transformation and deformation, or simply move objects on a virtual canvas, finally adjusting their location.

The transformation menu provides access to the following actions: increase and decrease, rotate, flip, tilt, warp, perspective distortion and curvature. Zooming in and out, combined with moving an object, allow you to make nuanced compositional changes that would be impossible or extremely difficult in a traditional drawing (for example, you would have to erase the object and draw anew in a pencil sketch) (Fig. 18). 

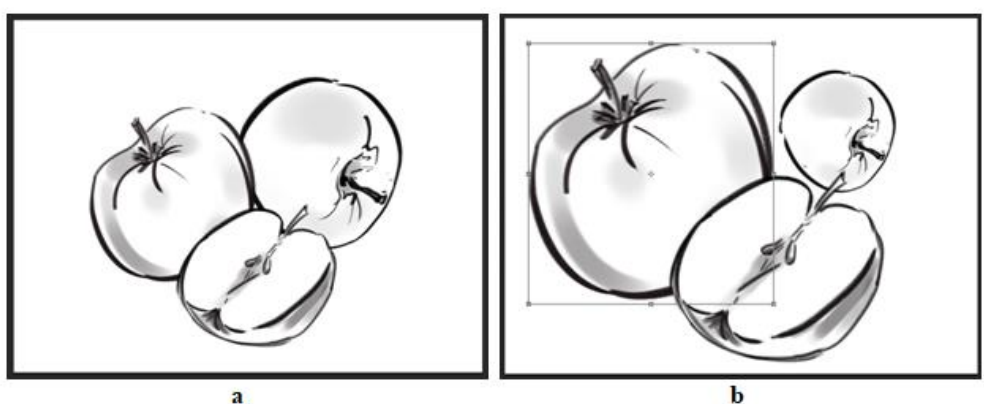

Fig. 18. Zooming: a) original composition; b) objects moved, resized, left apple in transform mode.

The rotation function allows you to rotate the object relative to the selected center (Fig. 19a). This can be done both with individual objects, and with all at the same time.

Moreover, rotation (like any other transformation) can be applied to any area of the object. The same applies to the mirror image of an object or its part.

"Tilt" is a warping function. The object is placed in a virtual quadrangular container, each of the corners of which moves horizontally or vertically, changing the geometry of the object (Fig. 19b).

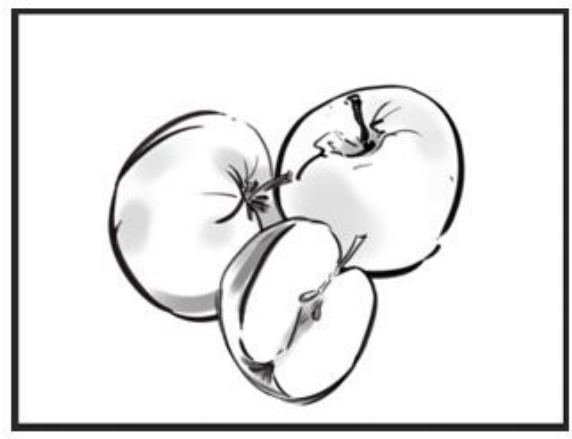

a

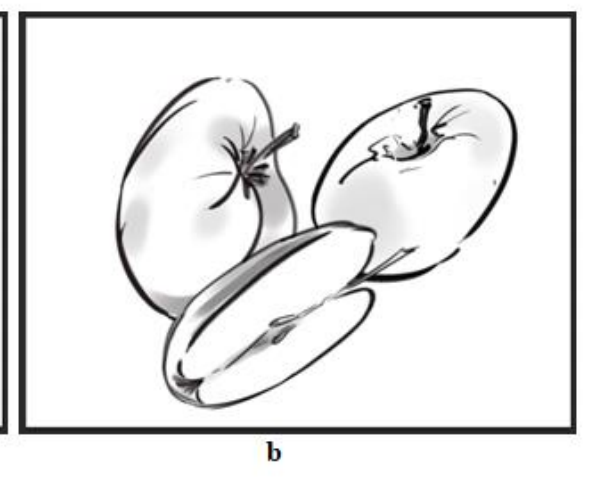

Fig. 19. a) Objects are deployed relative to each other b) tilt change.

The principle of the "Distortion" operation is similar to the "Tilt", with the difference that in the process of distortion the corner points of the transforming container can move not only horizontally or vertically, but also along diagonals (Fig. 20a). This makes the distortion function a more flexible tool.

In the transforming container "Perspectives", the two nearest corners have a mirror connection, allowing to make perspective distortion of objects with symmetric accuracy (Fig. 20b). At the stage of work on the composition, the artist often comes up with new ideas regarding the general concept, and they can be implemented in the form of new sketches; but in some cases it is more productive to use the previous versions, reworking them accordingly to 
the new idea. In such cases, the perspective transformation becomes the best choice, as it allows you to quickly transform objects under a new angle.

The next function, "Warp", places the object in a grid of nine cells (Fig. 20c). Of all the listed tools, this one is the most thorough and opens up wide possibilities for transforming an object, since the operations that modify an object are carried out not at the expense of external reference points, but occur "inside" the object. Of course, this kind of deformation significantly distorts both the object itself and its contours, so that it is suitable mainly for preliminary compositional sketching.

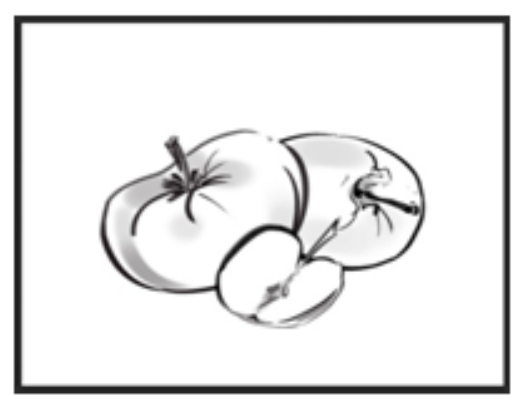

a

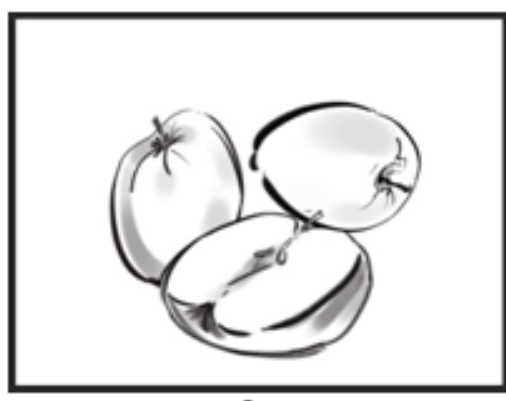

b

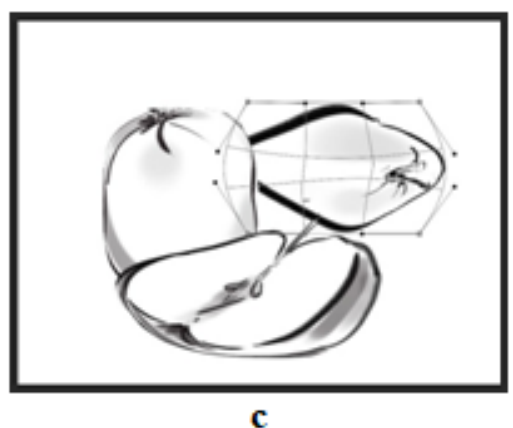

c

Fig. 20. Transformations: a) distortion; $b$ ) perspective; c) deformation.

The following tool has a more complex structure and principle of operation. In the Adobe Photoshop graphic editor, it has the name Puppet Warp (Fig. 21). A fine mesh is superimposed on the object; on the grid, the artist places the reference points, fixing them in places or making them moveable. Similar to the puppet's principle of operation, in which the joint nodes move relative to each other, the points in the transformed object are deformed according to the anchored points. With regard to the composition of the figure, such a functional allows you to change the position of the figure: for example, bend the hand of the depicted person in the elbow, make a bend of the tree.

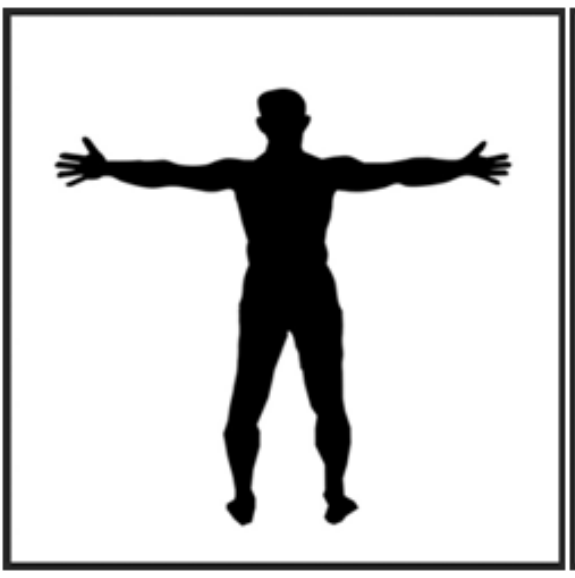

a

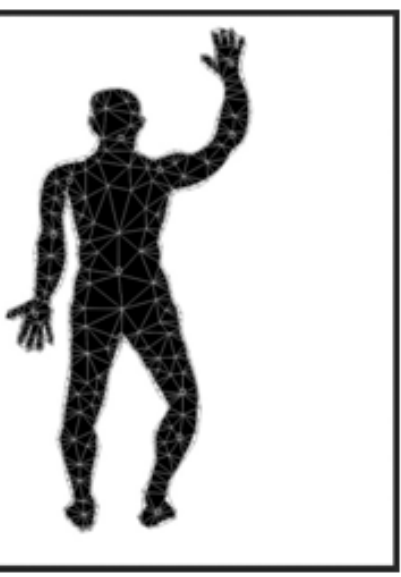

b

Fig. 21. a) original image of figure; b) changing the position of the figure using the "puppet warp". 
Building a composition, it is necessary to take into account some specific features of the digital drawing. Such images, as a rule, exist exclusively in digital representation, are exhibited in online galleries and distributed via the Internet. Therefore, the image format must meet the needs of the audience of digital artists. For example, so that the composition is located within the framework of such aspect ratio of the sheet, which as a result would allow using the image as wallpaper for a computer desktop (16: 9). Of course, this is not a mandatory requirement, but you should not neglect the requirements of the modern community of digital artists and fans of digital painting.

\section{References}

1. Toshio Ban The Osamu Tezuka Story: A Life in Manga and Anime. Stone Bridge Press, 2016. 928 p.

2. Mironov D. Komp'juternaja grafika $v$ dizajne [Computer graphics in design] // BHW Petersburg. 2008. 560 p. [in Russian].

3. Joline Blais, Jon Ippolito. At the Edge of Art. Thames \& Hudson. 2006. 256 p.

4. Perova Olga Fan-Art: Independent Art or Blind Copying? Young Scientist USA. 2018. No. 14. P. 31-36.

5. Summerer C. "Illegale Fans": Die urheberrechtliche Zulässigkeit von Fan Art. De Gruyter. 2015. 255 p.

6. Beginner's Guide to Digital Painting in Photoshop: Characters. 3d ed. 2015. 224 p. 